\title{
AN EXPERIMENTAL AND ANALYTICAL STUDY OF PLASMA CLOSING SWITCHES FILLED WITH ENVIRONMENTALLY FRIENDLY GASES
}

\author{
C. McGarvey, I. V. Timoshkin, S. J. MacGregor, M. P. Wilson, M. J. Given, \\ High Voltage Technologies, University of Strathclyde, Glasgow, United Kingdom \\ M. A. Sinclair \\ Hydrodynamics Division, AWE, Aldermaston, United Kingdom
}

\begin{abstract}
In recent years there has been a desire within the pulsed power community to find potential alternative gases to sulphur hexafluoride $\left(\mathrm{SF}_{6}\right)$ for use within pulsed power systems. Within plasma closing switches (PCSs), the desire to use environmentally friendly gases has come as a result of environmental concerns over the emissions of currently used gases into the atmosphere and contributing to the global warming problem. One of the main issues in finding a suitable replacement gas or gases for use in PCSs is that the performance characteristics of a switch filled with an alternative gas or gas mixture should be comparable to the performance characteristics of conventional $\mathrm{SF}_{6}$-filled switches.

The research presented in this paper is an expansion of previous work conducted and forms an experimental and analytical evaluation of breakdown characteristics in two commonly used PCS topologies (a two-electrode selfbreakdown switch and a field distortion switch) when filled with different gases (air, oxygen-nitrogen mixtures, argon oxygen mixture, nitrogen and carbon dioxide) over a range of pressures from $0.1 \mathrm{MPa}$ to $0.45 \mathrm{MPa}$ and for a range of inter-electrode distances.
\end{abstract}

\section{I.INTRODUCTION}

Despite the desirable properties of $\mathrm{SF}_{6}$ such as its high dielectric strength, voltage recovery capabilities and excellent insulating properties, $\mathrm{SF}_{6}$ is a greenhouse gas [1]. For this reason along with the continual rise in cost of obtaining $\mathrm{SF}_{6}$, many areas of the pulsed power industry desire to move away from $\mathrm{SF}_{6}$ usage and look into finding a suitable gas to replace $\mathrm{SF}_{6}$ in the HV pulsed power systems.

When trying to identify a potential replacement gas, one of the main challenges is that the gas in question should provide similar operational characteristics as conventional $\mathrm{SF}_{6}$-filled switches e.g. [3] the switches should be able to reliably reach required dc breakdown voltages, have a specified value of breakdown voltage spread, triggered breakdown voltages should be of similar levels to those currently in operation, jitter must be minimized and fall within operational requirements, recovery times of the switch as well as energy losses in the system should be comparable to the equivalent parameters obtained within currently used pulsed power machines.

There has been work conducted on other, high dielectric strength gases and there have been a number of gases identified, such as $\mathrm{C}_{3} \mathrm{~F}_{8}, \mathrm{CF}_{4}, \mathrm{CHF}_{3}$ [4] which under testing, have shown comparable or increased performance compared with $\mathrm{SF}_{6}$, and thus, could be considered in some cases as alternatives gases within PCSs. The main disadvantage of using these gases $\left(\mathrm{C}_{3} \mathrm{~F}_{8}, \mathrm{CF}_{4}, \mathrm{CHF}_{3}\right.$ or other strongly electronegative gases) is that they are strongly electronegative, many of them are fluorocarbons and so are also considered to be damaging to the environment. There has yet to be a gas or gas mixture identified which when filling a plasma closing switch provides comparable operational properties to the conventional $\mathrm{SF}_{6}$ filled plasma closing switches and that is also considered to be environmentally friendly.

Ideally oxygen, nitrogen [5] or some combination of the two gases under pressure could provide potential solution to the problem of replacing $\mathrm{SF}_{6}$. Not only are both of these gases not considered damaging to the environment, they are widely available and therefore are cheaper to obtain than $\mathrm{SF}_{6}$ so would provide a viable alternative for industrial applications requiring vast quantities of gas.

This paper builds on previously obtained experimental results [6][7] for DC breakdown tests conducted in a selfbreakdown switch with an adjustable inter-electrode gap which has been filled with argon, nitrogen, atmospheric air or an argon/oxygen mixture at different pressures. As a result of the experimental work conducted, certain operational characteristics of the plasma closing switches have been calculated and compared with the characteristics of commonly used $\mathrm{SF}_{6}$-filled switches.

\section{II.SELF-BEAKDOWN SWITCH}

\section{A. Design and Construction.}

In order to investigate operational performance of a switch filled with air, nitrogen, nitrogen/oxygen mixture and carbon dioxide, a self-breakdown switch was designed, modeled in electrostatic software and then constructed. The dimensions of the self-breakdown switch as well as the operational pressure requirements of 
the switch were established and modeled using electrostatic software QuickField before putting the switch into construction to ensure that there would be no unwanted field enhancement within the switch body during testing. The body of the switch was constructed from a black PVC cylinder with $102 \mathrm{~mm}$ internal diameter. The spherical electrodes are $16 \mathrm{~mm}$ diameter and made from brass. The inter-electrode gap spacing is adjustable in $1 \mathrm{~mm}$ increments, the maximum gap is $9 \mathrm{~mm}$. A $25 \mathrm{~mm}$ diameter quartz window was mounted into the switch body to allow for optical diagnostics of the plasma channel(s) formed during breakdown events. Gas is supplied to the switch via a gas distribution board and the test-cell has been pressure tested and showed no leakage of the gas from the test cell up to a maximum pressure of $0.5 \mathrm{MPa}$ in line with industrial operational requirements. 5 gases/gas mixtures have been tested throughout the experiments within this self-breakdown switch: air; nitrogen; $60 \% / 40 \%$ nitrogen /oxygen mixture;carbon dioxide $\left(\mathrm{CO}_{2}\right) ;$ and a $90 \% / 10 \%$ argon/oxygen mixture.

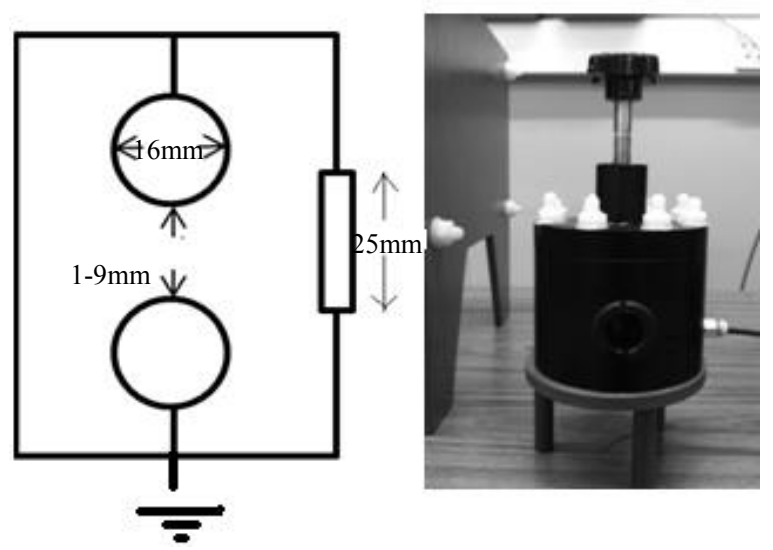

Figure 1. Schematic diagram and photograph of experimental self-breakdown switch design.

\section{B. Experimental Setup}

D.C. breakdown voltage experiments were carried out by setting-up a basic RLC circuit: a switch was connected in parallel to a $45 \mathrm{nF}$ capacitor and charged through a $100 \mathrm{k} \Omega$ charging resistor until breakdown occurs. An inductor was added into series with the switch. The capacitor and the switch were charged using a $100 \mathrm{kV}$ d.c. Glassman high voltage power supply. A North Star PVM-5 voltage probe was used to monitor the breakdown voltage and the breakdown current was monitored by the current shunt, voltage and current waveforms were registered by a Tektronix 2024 digital oscilloscope. The gases tested were supplied to the system via a gasdistribution board, which allowed for monitoring of the gas pressure within the system during evacuation and repressurising and ensured that no gas was leaking from the test cell during the experimental procedure.

The inter-electrode gap space in the switch was set to the required distance before the test-cell was evacuated by means of a vacuum pump before being filled with bottled air to $0.1 \mathrm{MPa}$. The top electrode was stressed with high voltage which was increased manually at a rate of $\sim 2 \mathrm{kV} / \mathrm{s}$ until breakdown event was on the oscilloscope. The bottom electrode was held at ground potential. 20 breakdown tests at the same gap-space and pressure were conducted before the gas in the test-cell was removed, again by use of a vacuum pump, and then filled with fresh gas at an increased pressure.

The experimental procedure outlined above was repeated for each gas/gas mixture, each inter-electrode distance (in the range $1 \mathrm{~mm}-9 \mathrm{~mm}$ ) and gas pressure within required operational range. The average value of breakdown voltage and its standard deviation were calculated for each combination of the gas, electrode gap and pressure.

\section{Self-Breakdown Voltage}

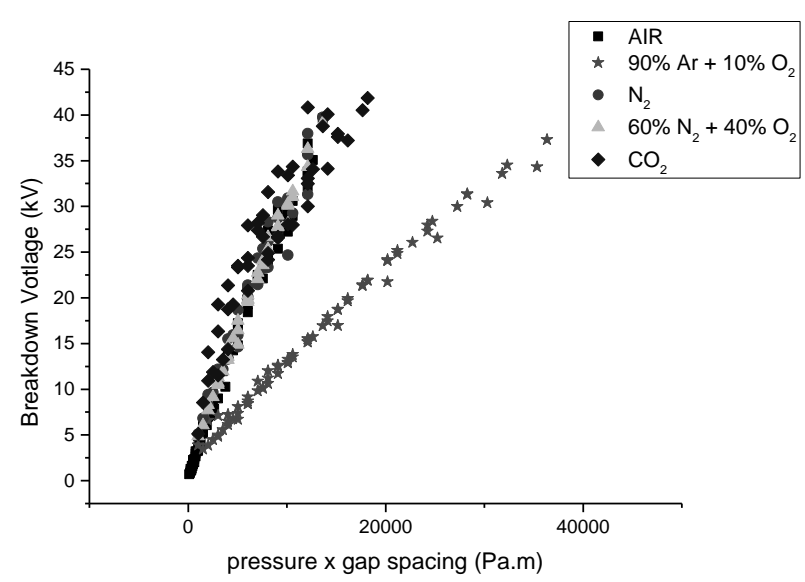

Figure 2. Self-breakdown voltage as a function of $p d$ the product of the pressure and the inter-electrode gap spacing in the switch.

The results of self-breakdown tests are presented in Figure 2. This Figure 2 shows the breakdown voltage behavior as a function of $p d$, the product of the gas pressure in the switch, $p$, and the inter-electrode gap space, $d$.

The experimental results show that the largest breakdown voltage capabilities came from the switch filled with $\mathrm{CO}_{2}$ and the lowest breakdown voltages achieved came when the switch was filled with the $90 \%$ argon $/ 10 \%$ oxygen mixture. When the switch was filled with air, nitrogen and the $60 \%$ nitrogen $/ 40 \%$ oxygen mixture, the breakdown voltages were largely similar to each other and close to that of the $\mathrm{CO}_{2}$ filled switch. The standard deviation in the breakdown voltages obtained decreased as product $p d$ increased for all gases. The largest standard deviation values came from the switch filled with $\mathrm{CO} 2$. The mixture of $60 \%$ nitrogen $-40 \%$ oxygen showed the lowest standard deviation in terms of breakdown voltage. 


\section{Plasma Temperature}

Table 1. Values of statistical weights and Einstein coefficients, [9].

\begin{tabular}{|l|l|l|l|l|l|l|}
\hline $\begin{array}{c}\text { Cu line } \\
\text { peak, } \\
\text { nm }\end{array}$ & 510 & $\mathbf{5 1 5}$ & $\mathbf{5 2 1}$ & $\mathbf{5 2 9}$ & $\mathbf{5 6 7}$ & $\mathbf{5 7 7}$ \\
\hline $\begin{array}{l}g A, 10^{-8}, \\
s^{-1}\end{array}$ & 0.051 & 4.7 & 5.8 & 3.2 & 0.014 & 0.054 \\
\hline$E, e V$ & 2.65 & 4.31 & 4.31 & 5.38 & 2.65 & 2.63 \\
\hline
\end{tabular}

Calculations of the temperature of the plasma channels formed during the breakdown process were conducted as a method of evaluating the energy deposited into the plasma channel during breakdown process. This will ultimately lead to the calculation of resistance of the plasma channel. The Boltzmann method [8] of calculating plasma temperature was followed.

Breakdown occurrences were created using the method described previously for each gap space, pressure and gas mixture. An Ocean Optics HR-4000 spectrometer was mounted in a holder connected to the quartz window installed in the side of the test-cell. For each breakdown occurrence, the light emission from the plasma (spark discharge) was registered using the Ocean Optics SpectraSuite software. 5 repeated breakdown tests for each variable parameter were conducted and the emission spectrum for each breakdown was obtained and analysed.

Analysis of the emission spectra allowed us to identify the emission lines of copper at $510 \mathrm{~nm}, 515 \mathrm{~nm}, 521 \mathrm{~nm}$, $529 \mathrm{~nm}, 567 \mathrm{~nm}$ and $570 \mathrm{~nm}$ wavelengths. The relative intensities of the peaks were noted and used in the equation

$$
\ln \left(\frac{\lambda I}{g A}\right)=C-\frac{E_{i}}{k T}
$$

where $\lambda$ is the wavelength of emission, $I$ the corresponding intensity of emission at that wavelength, $g$ is the statistical weight of the upper level, $A$ is Einstein's transitional probability, $E$ is the energy of the upper level of the transition, $k$ is Boltzmann's constant and $T$ is the absolute temperature of a source following a Bolzmann distribution of the energy. The values of $E$ and $g A$ are known for copper emission lines at the required wavelengths [8].

To calculate the temperature of the plasma formed in the switch during breakdown, the logarithmic term was plotted against the energy $E$. A line of best fit was added to the points and the gradient of this line determined the temperature of the plasma channel.

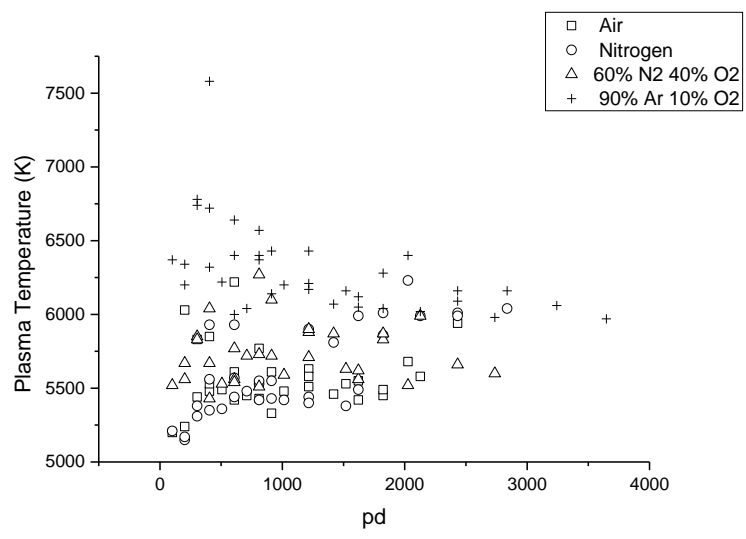

Figure 4. Plasma temperature for the tested gases at different values of $p d$..

As can be seen from Figure 3 above, the temperature of the plasma sparks formed tends to increase as $p d$ increases for 4 of the 5 gases tested. In the case of a switch filled with the $90 \%$ argon $/ 10 \%$ oxygen mixture, the temperature of the plasma channel was seen to decrease as the value $p d$ increased. The temperature of the plasma channels formed during breakdown lies within the range of $5000 \mathrm{~K}$ $-7000 \mathrm{~K}$ for all of the gases tested. The standard deviation of the temperature decreases as the product $p d$ increases for all gases.

\section{FIELD DISTORTION TRIGGERED SWITCH}

\section{A. Design and Construction}

The field-distortion triggered spark gap switch was constructed from a PVC body and also makes use of two spherical brass electrodes $16 \mathrm{~mm}$ in diameter, identical to those used in the elf-breakdown switch design. A midplane trigger electrode is located in the centre of the interelectrode gap and is constructed from a sheet of brass, $1 \mathrm{~mm}$ in thickness with an aperture cut from the centre.

The gap spacing between the top and bottom spherical electrodes is adjustable from $2 \mathrm{~mm}-10 \mathrm{~mm}$. The same gas distribution board as used in the self-breakdown test setup was used within the triggered switch test set-up for evacuation and refilling of the test cell with the gases under investigation.

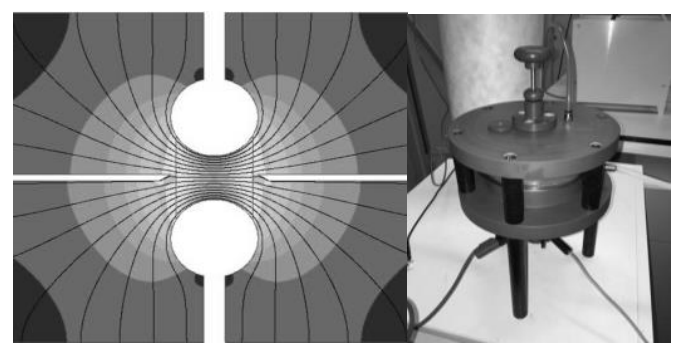

Figure 5. Field distribution in the field-distortion spark gap switch; constructed switch. 


\section{B. Experimental Setup}

The experimental setup of the triggered switch system is similar to that of the self-breakdown switch test setup. However, the triggered switch set-up also makes use of a trigger generator which outputs a $28 \mathrm{kV}$ negativevoltage impulse with microsecond rise-time.

For the results presented within this section, all experiments were conducted with $4 \mathrm{~mm}$ inter-electrode gap spacing. The gases tested were air, nitrogen and the $90 \%$ argon $/ 10 \%$ oxygen mixture. The pressures tested remained the same, $0.1 \mathrm{MPa}-0.45 \mathrm{MPa}$ range increasing in steps of $0.05 \mathrm{MPa}$.

\section{Results - Self Breakdown}

The self-breakdown voltage for the triggered switch topology was investigated as the maximum operational dc voltage of the triggered switch topology.

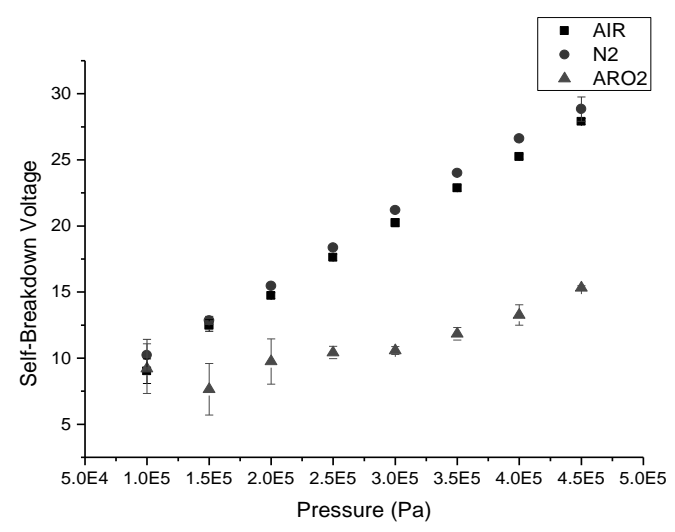

Figure 7. Self-breakdown voltage as a function of pressure.

The results presented in Figure 7 above show that for the three gases tested, there is an increase in the breakdown voltage within the switch as the pressure of the gas increases. For the air filled switch and the nitrogen filled switch, the voltage required to initiate breakdown increases almost linearly as pressure increases. For the switch filled with the $90 \%$ argon/ $10 \%$ oxygen mixture, the voltage required for initiating breakdown increases also, but it is not a linear increase as pressure increases.

\section{Results - Trigger pulse - No DC Stress}

For the results presented here, it was investigated whether it would be possible to initiate breakdown within the switch by applying a trigger pulse to the mid-plane electrode and grounding both the top and bottom spherical electrodes.

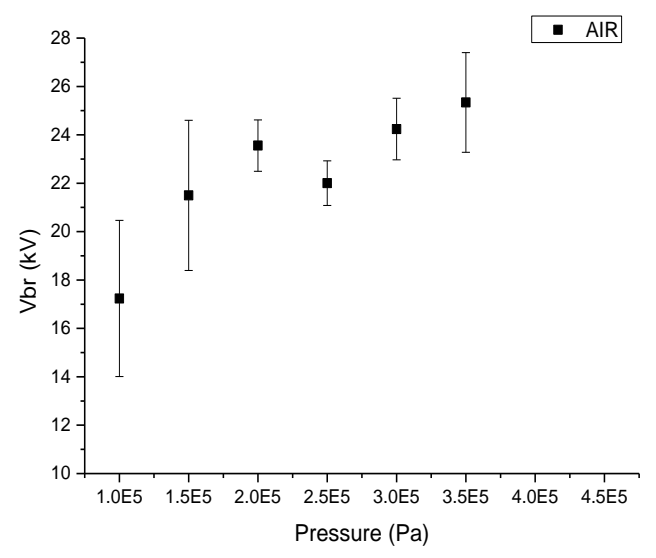

Figure 8a. Trigger voltage required to initiate breakdown in an air-filled switch as pressure increases.

As can be seen from Figure 8a. above, the trigger voltage required to initiate breakdown under the conditions described within an air-filled switch increases in a nonlinear fashion as the gas pressure within the switch increases. The standard deviation in the voltage required to initiate breakdown was seen to decrease from $3.3 \mathrm{kV}$ maximum standard deviation to a minimum of $0.9 \mathrm{kV}$ with increasing pressure and then increase slightly upon further increase of pressure.

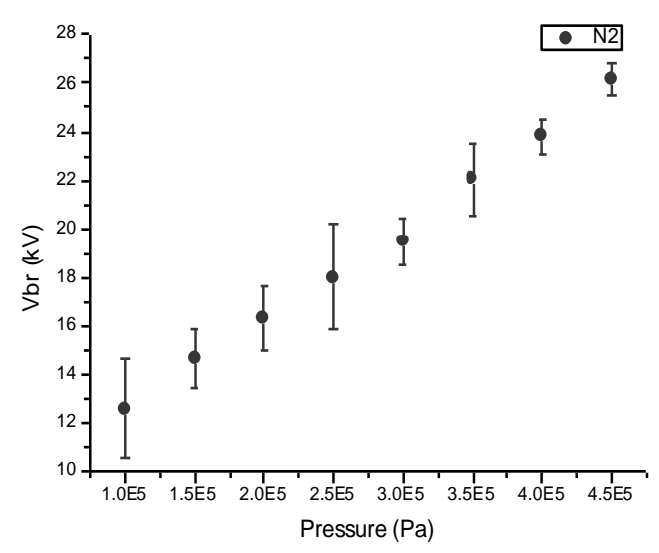

Figure 8b. Trigger voltage required to initiate breakdown in a nitrogen filled switch as pressure increases.

Figure $8 \mathrm{~b}$. shows that the trigger voltage required to initiate breakdown in a nitrogen filled switch under impulsive triggering switch increases in a linear fashion as the gas pressure within the switch increases. The standard deviation in the voltage required to initiate breakdown was seen to be unstable although generally decreases from $2 \mathrm{kV}$ to $0.6 \mathrm{kV}$ as pressure increases. 


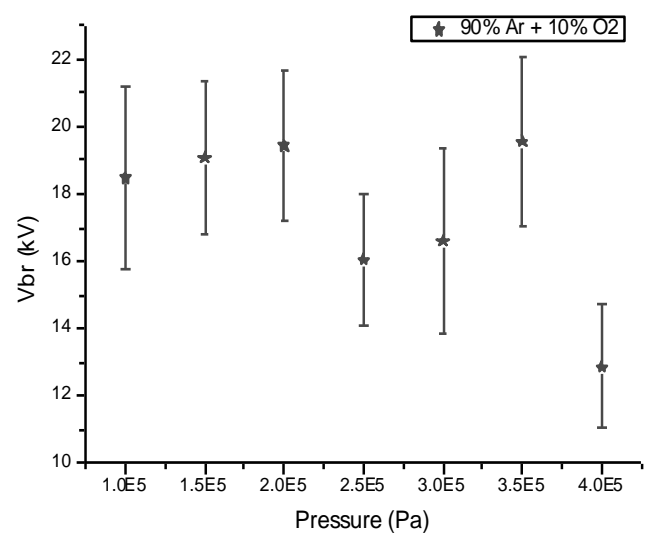

Figure 8c. Trigger voltage required to initiate breakdown in a switch filled with the $90 \% / 10 \%$ argon/oxygen mixture as pressure increases.

Figure 8c. shows the behavior of trigger voltage required to initiate breakdown in a switch filled with the $90 \%$ argon $/ 10 \%$ oxygen mixture. For the voltage impulse required to initiate breakdown in this switch was actually seen to decrease as pressure increased. For all gas pressures tested, the standard deviation in breakdown voltage was large, typically $2.6-2.8 \mathrm{kV}$.

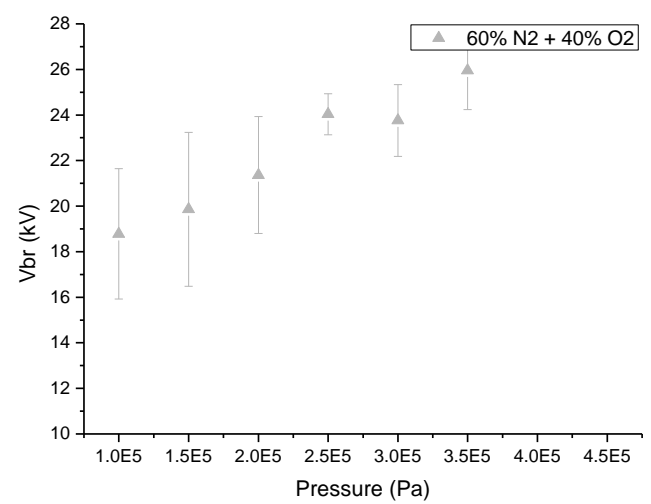

Figure 8d. Self-breakdown voltage as a function of $p d$.

Figure $8 \mathrm{~d}$ shows the behavior of trigger voltage required to initiate breakdown in a switch filled with the $60 \%$ nitrogen $/ 40 \%$ oxygen mixture. As was seen in the case of the air-filled switch, the voltage required for breakdown increases in a nonlinear fashion as pressure increases. The standard deviation in the nitrogen-oxygen mixture was also seen to decrease from $3 \mathrm{kV}$ to $0.9 \mathrm{kV}$ before increasing again as the pressure of the gas within the switch increased.

In the case of the switch filled with carbon dioxide, no breakdown was seen to occur above $0.1 \mathrm{MPa}$ and thus the results have been omitted from this publication.

\section{E. Results - DC stress \& trigger pulse}

Once the investigation of triggered breakdown in the absence of dc stress voltage had been conducted, the investigation of the breakdown voltage when a dc stress voltage was applied to the upper spherical electrode was conducted. The experiments were conducted with three different gases; air, nitrogen and the $90 \%$ argon $/ 10 \%$ oxygen.

The dc stress voltage was applied to the top spherical electrode while the bottom spherical electrode was grounded. The de stress voltage was held at $80 \%$ of the self-breakdown voltage for both the air and nitrogen filled switches. In the case of the $90 \% / 10 \%$ argon/oxygen mixture filled switch, the dc stress level was held at $70 \%$ due to self-breakdown occurring regularly at the $80 \%$ stress level. (a)

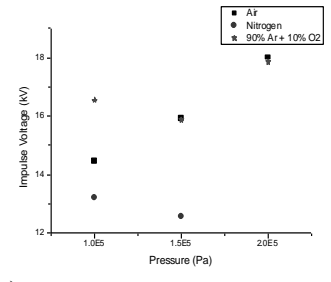

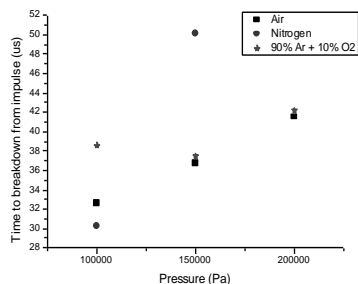

(b)
Figure 9. Self-breakdown voltage as a function of $p d$..

Figure 9a shows that when an $80 \%$ stress level is applied, the voltage impulse (applied to the mid-plane electrode) required to initiate a breakdown in an air-filled switch increases linearly as pressure increases. In the case of a nitrogen filled switch, the value was seen to decrease, however, breakdown was not see to occur at pressures $0.2 \mathrm{MPa}$ or above. In the switch filled with the $90 \%$ argon and $10 \%$ oxygen mixture, the voltage required to initiate breakdown appeared to decrease before increasing.

Figure 10. Self-breakdown voltage as a function of $p d$..

Figure $9 \mathrm{~b}$ shows the time taken to breakdown for the field-distortion spark gap switch. The time taken to breakdown is defined as the time from the initiation of the voltage pulse until the breakdown event was seen to occur. As expected, and in line with the increase in voltage required to cause breakdown in the switch, the time taken between the initiation of the voltage pulse and breakdown occurring generally was seen to increase for all three gases as pressure increased. However, because of the limited range of pressures tested and the lack of results at pressures of $0.2 \mathrm{MPa}$ and above for nitrogen, further investigation must be conducted.

\section{CONCLUSIONS}

The results presented within this paper form an experimental investigation of two commonly used switch 
topologies when filled with environmentally friendly gases.

A self-breakdown switch with similar dimensions and operational pressure capabilities has been designed, constructed and filled with different gasses at a range of pressures. The highest breakdown voltage was obtained when the switch was filled with $\mathrm{CO}_{2}$ although this did also provide the largest standard deviation in breakdown voltages. The maximum standard deviation seen came from a $\mathrm{CO}_{2}$ filled switch to $0.1 \mathrm{MPa}$ when the standard deviation in breakdown voltage was $2.4 \mathrm{kV}$. The lowest breakdown voltage capabilities came from the switch when filled with the $90 \%$ argon $/ 10 \%$ oxygen mixture. For all gases, the breakdown voltage increased as the product $p d$ increased and in all cases, the standard deviation in breakdown voltage decreases as $p d$ increases with the smallest standard deviation in breakdown voltage being obtained in the switch filled with the $60 \%$ nitrogen $-40 \%$ oxygen mixture to $0.15 \mathrm{MPa}$ where the standard deviation was $0.01 \mathrm{kV}$.

The temperature of the plasma sparks formed during breakdown has been obtained by analyzing emissions of light and applying the Boltzmann approach. The temperature of the plasma lie in the region of $5000 \mathrm{~K}-$ $7000 \mathrm{~K}$ for all gases and with the exception of the argonoxygen mixture where the temperature of the plasma channel was seen to decrease as $p d$ increases, all other gases showed an increase in the temperature of the plasma channels as $p d$ increased.

A field-distortion triggered switch has also designed and constructed with similar dimensions and operational requirements to those currently used within industry. For all conducted experiments within this field-distortion spark gap switch, the inter-electrode gap space was held at $4 \mathrm{~mm}$

The self-breakdown voltages within the switch when filled with three gases at a range of pressures have been obtained as the upper limit of DC stress voltage capabilities. Nitrogen and air-filled switches showed a linear increase in breakdown voltage as pressure increases. The $90 \%$ argon/ $10 \%$ oxygen mix also showed an increase in breakdown voltage as pressure increases, although not a linear increase.

The triggered breakdown voltage in the absence of a dc stress voltage showed a linear increase in voltage required to initiate breakdown when the switch was filled with nitrogen. A nonlinear increase in the voltage required to initiate breakdown was observed in switches filled with the $60 \%$ nitrogen and $40 \%$ oxygen and the air-filled switch. In the case of the switch when filled with the $90 \%$ argon and $10 \%$ oxygen mixture, the triggered voltage required to initiate breakdown was seen to decrease as pressure increased.

A dc stress voltage of $80 \%$ of the self-breakdown voltage was then applied to the top spherical electrode (70\% in the case of the argon-oxygen mixture) before the trigger pulse was applied to the mid-plane electrode. The air-filled gap shows a linear increase in impulse voltage required to initiate breakdown as pressure increases. The nitrogen filled gap appeared to show a decrease in impulse voltage required to initiate breakdown as pressure increased however no breakdown was seen to occur under these test conditions for pressures $0.2 \mathrm{MPa}$ or above and thus further work must be conducted. The argon-oxygen mixture filled gaps generally show an increase in impulse voltage required and time required before breakdown occurs. In all cases, the standard deviation in breakdown voltage decreased typically from $2.5 \mathrm{kV}$ to $1.8 \mathrm{kV}$ as pressure increased.

\section{V.FUTURE WORK}

The experimental results obtained will be expanded upon and will be used to develop a computational model that will accurately describe the operation of currently employed pulsed power systems.

The model will allow for characterisation of future pulsed power devices as well as optimisation of current and future pulsed power systems.

Further experimental work will be conducted with the triggered switch to gain further detailed results for use in developing the computational model e.g. resistance of the plasma channel(s) formed during breakdown, energy losses in the switch and inductance of the system.

Results obtained from filling the designed and constructed switches with environmentally friendlier gases than $\mathrm{SF}_{6}$ will be compared to conventional $\mathrm{SF}_{6}$ filled switches.

\section{REFERENCES}

[1] United Nations Framework on Climate Change: Global Warming Potentials http://unfccc.int/ghg_data/items/3825.php

[2] United Nations Framework on Climae Change: Kyoto Protocol http://unfccc.int/kyoto_protocol/items/2830.php

[3] J. Mankowski, M. Kristiansen, "A review of Short Pulse Generator Technology" IEEE Transactions on Plasma Science, v.28, pp.102-108, 2000

[4] "Gases for electrical insulation and arc interruption: possible present and future alternatives to pure $\mathrm{SF}_{6}$ "

[5] Suitability of nitrogen to replace $\mathrm{SF}_{6}$

[6] C.McGarvey, I.V. Timoshkin, S.J. MacGregor, M.P. Wilson, M.J. Given, M.A. Sinclair "Breakdown Characteristics of Plasma Closing Switches filled with different gases" $20^{\text {th }}$ International Conference on Gas Discharges and their Applications, Orleans, France July $6^{\text {th }}-13^{\text {th }} 2014$.

[7] C.McGarvey, I.V. Timoshkin, S.J. MacGregor, M.P. Wilson, M.J. Given, M.A. Sinclair "Characterisation of a plasma closing switch filled with environmentally friendly gases" Pulsed Power Conference (PPC), 2013 19th IEEE, 1-5

[8] C. H. Corliss "Experimental Transitional Probabilities for spectral lines of seventy elements" U.S. Department of Commerce, 1962

[9] www.nist.gov 